Objectives: To study the translation and cultural adaptations of the OA Guidebook appropriate for local context and use by patient champions and health professionals together

Methods: A project launch meeting led to the creation of an international panel of Patient Champions and the adaptation of the OA Guidebook as a priority project. 15 Patient Champions with OA were collaborative partners of the project's local Communities of Practices (CoP) (UK:2, Norway:2, Netherlands:5, Denmark:5, Portugal:1) (figure 1). CoPs also engaged with OA patient organisations. Cultural adaptation of the OA Guidebook by CoPs: 1) review of UK OA Guidebook and existing written patient information; 2) translation; 3) cultural adaptation: review of content, images and layout; 4) consistency check with national guidelines; 5) production; 6) review and approval; 7) shared learning across countries via Skype. Each CoP adopted a process appropriate to their specific context Results:

Abstract OP0346-PARE - Table 1 Development of culturally adapted versions of the OA Guidebook in each country.

\begin{tabular}{lc}
\hline Country & Developments to date \\
\hline Netherlands & $\begin{array}{c}\text { The Dutch team produced a full version of the OA Guidebook, revised the } \\
\text { translated text and recommended new images to better reflect Dutch } \\
\text { patients. Now available in hard copy and on a OA patient website: www.poly- } \\
\text { artrose.nl/ }\end{array}$ \\
Norway & $\begin{array}{c}\text { The Norwegian CoP supplemented an existing OA handbook ('ActiveA') with } \\
\text { a 'Feeling Positive' chapter from the UK version. The Patient Champions } \\
\text { commented on the new chapter which will be made available on the } \\
\text { JIGSAW-E website. }\end{array}$ \\
Denmark & The Danish team produced a concise version of the OA Guidebook, \\
& containing vital written information for patients at first consultation. The \\
Portugal & The Portuguese team are working with the local health administration and \\
& patient organisations to culturally adapt a full Portuguese translation of OA
\end{tabular}
Guidebook

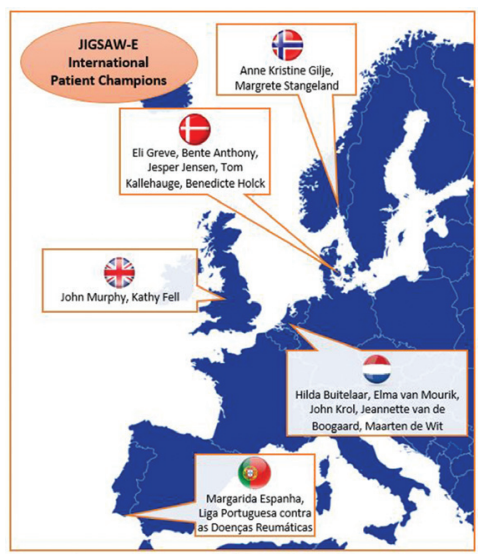

Abstract OP0346-PARE - Figure 1 The JIGSAW-E international panel of patient champions

Conclusions: It is feasible and effective to involve patient champions in the cultural adaptation and dissemination of consistent and accurate patient information to support the implementation of OA guidelines in clinical practice.

REFERENCES:

[1] ] Health Expect 2014;17:164-73.

[2] ] BMC Musculoskelet Disord 2014;15:427

[3] ] Osteoarthritis Cartilage 2018;26:43-53.

[4] ] Physiotherapy 2016;102:e138-9.

Acknowledgements: Thank you to all the Patient Champions for their contributions to JIGSAW-E. A. Rosager and L. Baumbasch led the Danish adaptation. JIGSAW-E is funded by EIT Health. KD is part funded by a NIHR Knowledge Mobilisation Research Fellowship (KMRF201403002). The MOSAICS study was funded by NIHR Programme Grant (RP-PG-0407-10386).

Disclosure of Interest: None declared

DOI: 10.1136/annrheumdis-2018-eular.6345

\section{OP0347 \\ SHARE DECISION MAKING AID FOR JUVENILE IDIOPATHIC ARTHRITIS: MOVING FROM INFORMATIVE PATIENT EDUCATION TO INTERACTIVE CRITICAL THINKING}

Y. El Miedany ${ }^{1,2}$, H. Lotfy ${ }^{3}$, N. El Aroussy ${ }^{1}$, D. Mekkawy ${ }^{1}$, S.I. Nasef ${ }^{4}$, W. Hassan ${ }^{5}$, Y. Farag $^{3}$, S. Almedany ${ }^{6}$, G. El Deriny ${ }^{7}$, M. Eissa ${ }^{8}$, M. El Gaafary ${ }^{9}$, on behalf of PRINTO Egypt. ${ }^{1}$ Rheumatology and Rehabilitation, School of Medicine Ain Shams University, Cairo, Egypt, ${ }^{2}$ Rheumatology, Darent Valley Hospital, Dartford, UK;

${ }^{3}$ Pediatrics, School of Medicine Cairo University, Cairo; ${ }^{4}$ Rheumatology and Rehabilitation, School of Medicine Suez Canal University, Ismaillia; ${ }^{5}$ Rheumatology and Rehabilitation, School of Medicine Banha University, Banha; ${ }^{6}$ Rheumatology and Rehabilitation, School of Medicine Tanta University, Tanta; ${ }^{7}$ Pediatrics, School of Medicine Alexandria University, Alexandria; ${ }^{8}$ Rheumatology and Rehabilitation, School of Medicine Cairo University; ${ }^{9}$ Community and Public Health, School of Medicine Ain Shams University, Cairo, Egypt

Background: Shared decision making (SDM) is an emerging trend in paediatrics. Currently available SDM interventions are mere information on the disease or medication and often fail to engage the children in the medical decision process. It has been suggested that future decision aids should consider developing separate more appropriate tools in order to better engage the children.

Objectives: To develop, and evaluate an illustrated and interactive evidencebased SDM aid for children living with inflammatory arthritis, able to inform them about the pros and cons of their disease as well as the available treatment options, and help them to make an informed shared decision.

Methods: A multidisciplinary team defined criteria for the SDM as to design, medical content and functionality, for children. Development was according to the international standard (IPDAS). Eight categories emerged as highly important for shared decision making: 1 . What is arthritis; 2 . Why do we treat arthritis; 3 . What are my targets?; 4 . What are the available treatment options?; 5 . Progressometer/ my chances of improvement; 6 . How soon will the medications kick in and how to take them; 7. Potential side effects; 8 . For how long shall I take the medication. Each category is supported by simple illustrations showing the state of the joint in the different scenarios whether the child take treatment or not, whereas at the end of each category the child is asked to make a decision in view of the information given. 94 children with juvenile idiopathic arthritis, evaluated the tool, in a randomised controlled study, in comparison to control group composed of 95 patients treated according to standard protocols.

Results: The shared decision making aid was developed to offer information about the disease, the risks and benefits of treatment. $97.5 \%$ of the children included reported comprehensibility of $>90 / 100$. the progressometer helped the children identify the importance of taking treatment for their disease. The patients adherence to anti-rheumatic therapy was significantly $(p<0.01)$ higher in the SDM group, whereas stopping DMARDs for intolerability was significantly $(p<0.01)$ higher in the control group at 12 months of treatment. There was significant improvement in the functional ability as well as quality of life measures in the SDM group $(p<0.01)$, whilst absence from school was significantly higher in the control group $(p<0.01)$

Conclusions: This Illustrated-interactive SDM aid for children living with idiopathic inflammatory arthritis was found to be a simple, user-friendly tool which can be implemented in standard clinical practice. The illustration and interactive style made it more attractive to the children. The developed SDM offered the children evidence-based information about the pros and cons of treatment options, improved their understanding of the disease, communication with their treating clinician as well as their ability to make an informed decision.

Disclosure of Interest: None declared

DOI: 10.1136/annrheumdis-2018-eular.2792

FRIDAY, 15 JUNE 2018

High-end imaging: Looking for the invisible

\begin{tabular}{|l|l}
\hline OP0348 & MASS SPECTROMETRY IMAGING ANALYSIS OF \\
SYNOVIUM DIFFERENTIATE PATIENTS WITH \\
PSORIATIC AND RHEUMATOID ARTHRITIS
\end{tabular}

B. Rocha ${ }^{1}$, B. Cillero-Pastor ${ }^{2}$, C. Ruiz-Romero ${ }^{1}$, A. Cuervo ${ }^{3}$, R.M. Heeren ${ }^{2}$, J. D. Cañete ${ }^{3}$, F.J. Blanco ${ }^{1}{ }^{1}$ Proteomics Unit-ProteoRed/ISCIII, Rheumatology Research Group, Inibic-C. Hospitalario Universitario A Coruña, A Coruña, Spain;

${ }^{2}$ The Maastricht Multimodal Molecular Imaging Institute (M4I), Division of MSI, Maastricht University, Maastricht, Netherlands; ${ }^{3}$ Arthritis Unit, Rheumatology Department, Hospital Clinic, IDIBAPS, Barcelona, Spain

Background: Rheumatoid arthritis (RA) and Psoriatic arthritis (PsA) are systemic inflammatory diseases characterised by a chronic form of arthritis, often leading to 
irreversible joint damage. Both are highly heterogeneous and complex disorders, presenting major challenges in diagnosis and treatment. The signs and symptoms of RA and PsA are similar especially at the earlier phases of the disease, so it can be difficult to distinguish them on clinical grounds. Lipids and metabolites have been associated with pathological events in both diseases as contributors of the inflammation process. Accordingly, the local lipidome and metabolome from the inflamed tissue may be more reliable in predicting the disease status than the current diagnostic methods, since the synovium is one of the main target tissues of both pathologies.

Objectives: To identify lipid and metabolic profiles in the synovium using Mass Spectrometry Imaging (MSI) that would have the potential to distinguish between patients with RA and PsA

Methods: Synovium biopsies from 25 patients with PsA, 21 with RA (16 seropositive and 5 seronegative) and 10 with IA (Indeterminate arthritis) were included Tissue sections were deposited on conductive slides and coated with different matrices for lipid and metabolite extraction. MALDI images were acquired on a rapifleX MALDI Tissuetyper time-of-flight instrument. Multivariate data analysis was used to look for the lipids and metabolites with the highest differences among groups.

Results: MALDI-MSI revealed differential lipid and metabolic profiles among all compared groups. Discriminant analysis (DA) performed on lipid data acquired in positive ion mode displayed a good separation of patients with PSA and RA, especially seropositive RA (figure 1A). PsA showed higher lipid content, mainly phospholipids and sphingolipids, compared to seropositive RA (figure 1B and 1C). Some of them showed a specific localization within tissue. Experiments performed in negative ion mode showed that phosphatidylinositols and phosphatidic acids content varied among groups. Accordingly, DA allowed the separation of PsA from RA and IA patients, mainly from seronegative RA. PsA and RA groups were also distinguished based on synovium metabolic signatures. Sugars including $N$ acetylhexosamine 6-sulfate $(\mathrm{m} / \mathrm{z} 282.0276)$, glucuronic acid 1-phosphate $(\mathrm{m} / \mathrm{z}$ 273.0026) and $N$-acetylneuraminic acid ( $\mathrm{m} / z$ 290.0876) displayed a stronger intensity in RA synovium when compared to PsA.

A)

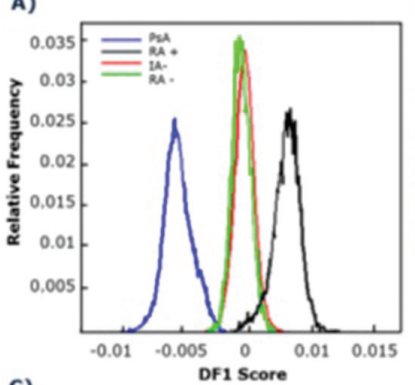

B)

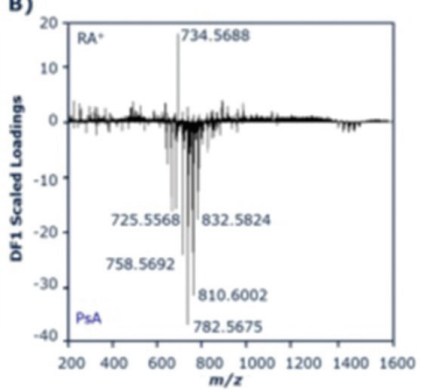

C

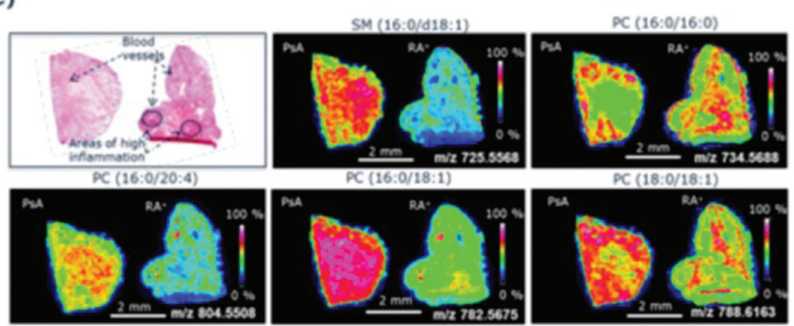

Abstract OP0348 - Figure 1. A) Histogram distribution and B) Discriminant function 1 (DF1) loading plot. DF1 negative scores are specific to PsA synovial tissue and positive scores to seropositive RA. C) Spatial mapping positive-lipid ions in synovium sections. Scale bar shows normalised intensities. Conclusions: For the first time, PsA and RA synovial membranes have been classified by MALDI-MSI. PsA synovium was characterised by a higher content of phospholipids compared to seronegative and seropositive RA. However, sugar metabolites displayed a stronger intensity in RA synovium when compared to PsA. Metabolic and lipid signatures here reported could support clinical decision-making in the diagnosis of RA and PsA.

Disclosure of Interest: None declared

DOI: 10.1136/annrheumdis-2018-eular.4466
SATURDAY, 16 JUNE 2018

\section{RA: is it all in your head?}

\section{OP0349 REMAINING PAIN AND WIDESPREAD PAIN DISTRIBUTION DURING THE FIRST 12 MONTHS AFTER RA DIAGNOSIS}

Y.C. Lee ${ }^{1}$, O. Schieir ${ }^{2}$, M.-F. Valois ${ }^{3}$, S.J. Bartlett ${ }^{3}$, G. Boire ${ }^{4}$, B. Haraoui ${ }^{5}$, C. Hitchon ${ }^{6}$, E. Keystone ${ }^{7}$, D. Tin ${ }^{8}$, C. Thorne ${ }^{9}$, J. Pope ${ }^{10}$, V. Bykerk $^{7,11}$, on behalf of CATCH. ${ }^{1}$ Northwestern University Feinberg School of Medicine, Chicago, IL, USA; ${ }^{2}$ University of Toronto Dalla Lana School of Public Health, Toronto; ${ }^{3}$ McGill University, Montreal; ${ }^{4}$ Universite De Sherbrooke, Sherbrooke; ${ }^{5}$ Institut de Rheumatologie de Montreal, Montreal; ${ }^{6}$ University of Manitoba, Winnipeg; ${ }^{7}$ University of Toronto, Toronto; ${ }^{8}$ Southlake Regional Health Center, Newmark; ${ }^{9}$ Southlake Regional Health Center, Newmarket, ${ }^{10}$ Western University, London, Canada; ${ }^{11}$ Hospital for Special Surgery, New York, NY, USA

Background: The incidence of fibromyalgia (FM), a chronic widespread pain syndrome, is highest in the first year after RA diagnosis. ${ }^{1}$ These 12 months may represent a critical window during which acute inflammatory pain transitions to chronic non-inflammatory pain. To prevent this transition, more data are needed about the course and predictors of pain during this time.

Objectives: 1) To describe the evolution of pain during the 12 months after RA diagnosis, and 2) to identify predictors of remaining pain and widespread pain (previously described as 'fibromyalgic RA') ${ }^{2}$ at 12 months.

Methods: Data were obtained from early RA patients in the Canadian Early Arthritis Cohort (CATCH), a prospective inception cohort. Primary outcomes were: 1) remaining pain above the Patient Acceptable Symptom State (PASS), defined as $4 / 10$ on a pain intensity numerical rating scale ${ }^{3}$ and 2 ) widespread pain, defined by tender joint count (TJC28) - swollen joint count (SJC28) $\geq 7 .^{2}$ Descriptive statistics were used to summarise the frequency of remaining pain and widespread pain over 12 months. Logistic regression models were used to identify predictors of remaining pain and widespread pain at 12 months. Variables forced into the multivariable models included age, sex, SJC28, ESR, depression, back pain, OA, sleep problems, HAQ-disability index (DI), MTX use, non-MTX conventional synthetic DMARD (csDMARD) use and NSAID use. Additional variables considered for inclusion via a backward selection process were race, education, income, FM, number of comorbidities and steroid use. Both models were adjusted for their respective baseline values.

Results: 1270 patients were included, with mean (SD) age of 53.9 (14.5) years, symptom duration of 5.8 (3.0) months and baseline DAS28 of 5.0 (1.4). The percentage of patients with remaining pain decreased from $64 \%$ at baseline to $24 \%$ at 12 months. The percentage of patients with widespread pain decreased from $9 \%$ to $5 \%$. The strongest predictors of 12 month remaining pain were sleep problems (highest quartile OR $2.2,95 \% \mathrm{Cl}: 1.2$ to 3.9 ), pain intensity $>4 / 10$ (OR 2.1 , $95 \% \mathrm{Cl}: 1.3$ to 3.4 ) and higher HAQ-DI score (OR $1.5,95 \% \mathrm{Cl}: 1.1$ to 2.0 ). The strongest predictors of 12 month widespread pain were higher HAQ-DI score (OR 1.8, 95\% Cl: 1.1 to 3.1 ) and higher number of comorbidities (OR 1.2, 95\% Cl: 1.0 to 1.5). Baseline non-MTX csDMARD use was associated with lower likelihood of widespread pain (OR $0.5,95 \% \mathrm{Cl}: 0.3$ to 0.8$)$.

Abstract OP0349 - Table 1 Multivariable logistic regression models for the association between baseline characteristics and a) remaining pain (pain NRS $>4$ ), and b) widespread pain distribution (TJC28-SJC28 $\geq 7$ )

\begin{tabular}{|c|c|c|}
\hline Baseline characteristics & $\begin{array}{l}\text { Remaining Pain }^{1} \\
\text { OR }(95 \% \mathrm{CI})\end{array}$ & $\begin{array}{c}\text { Widespread pain } \\
\text { OR }(95 \% \mathrm{Cl}) \\
\end{array}$ \\
\hline Age (for change of $10 \mathrm{yrs}$ ) & $0.8(0.7,1.0)$ & $0.7(0.6,0.9)$ \\
\hline Baseline pain intensity > 4/10 & $2.1(1.3,3.4)$ & $2.1(0.8,5.4)$ \\
\hline \multicolumn{3}{|l|}{ Sleep problems, quartiles } \\
\hline$\geq 0$ and $\leq 2$ & REF & REF \\
\hline$>2$ and $\leq 5$ & $1.8(1.1,3.1)$ & $0.7(0.3,1.9)$ \\
\hline$>5$ and $\leq 8$ & $1.8(1.1,3.0)$ & $0.9(0.4,2.2)$ \\
\hline$>8$ and $\leq 10$ & $2.2(1.2,3.9)$ & $1.0(0.3,2.7)$ \\
\hline Missing & $0.6(0.1,5.6)$ & $2.2(0.2,24.3)$ \\
\hline HAQ-DI & $1.5(1.1,2.0)$ & $1.8(1.1,3.1)$ \\
\hline Number of comorbidities & $1.1(1.0,1.3)$ & $1.2(1.0,1.5)$ \\
\hline Non-MTX csDMARD use & $0.8(0.6,1.1)$ & $0.5(0.3,0.8)$ \\
\hline
\end{tabular}

Conclusions: Despite improvements in pain during the first year after RA diagnosis, $24 \%$ continued to report remaining pain above the PASS, and $5 \%$ reported widespread pain at 12 months. Patients at greatest risk for 12 month remaining pain were those with sleep problems, severe pain and disability at baseline Patients at greatest risk for widespread pain were those with high baseline disability and many comorbidities. Baseline inflammation was not associated with 12 month pain outcomes.

REFERENCES:

[1] Lee YC, et al. Ann Rheum Dis 2013;72:649-54.

[2] Pollard LC, et al. Rheumatology 2010;49:924-8.

[3] Tubach F, et al. Arthritis Care Res 2012;64:1699-707. 